\title{
Cardiovascular magnetic resonance myocardial perfusion: methods and applications in patients with coronary artery disease
}

Authors: Chiara Bucciarelli-Ducci MD, Jonathan C. Lyne MRCP,

Peter D. Gatehouse PhD, Dudley J. Pennell FRCP FACC FESC

Chiara Bucciarelli-Ducci MD

Dudley J. Pennell FRCP FACC FESC

National Heart and Lung Institute, Imperial College, London, United Kingdom

Chiara Bucciarelli-Ducci MD, Jonathan C. Lyne MRCP, Peter D. Gatehouse PhD, Dudley J. Pennell FRCP FACC FESC

CMR Unit, Royal Brompton Hospital, London, United Kingdom

Address for correspondence:

Dudley J. Pennell

CMR Unit, Royal Brompton Hospital

Sydney Street

London, SW3 3TT

Email:

d.pennell@ic.ac.uk

ABSTRACT Perfusion CMR is an attractive imaging modality that is becoming comparable with other clinically diagnostic tests. SPECT and PET are well clinically validated and have good accuracy for detection of significant CAD. However, these techniques have a rather low spatial resolution and are not suitable for the detection of subendocardial perfusion defect. In addition, the radiation burden, the potential for attenuation artefacts (SPECT) and the limited availability (PET) are limitations of these imaging techniques. An integrated assessment of myocardial perfusion, function and viability is feasible with CMR. In addition, compared to other clinically available imaging techniques, CMR perfusion has excellent spatial resolution and no ionising radiation exposure. However, it is not widely available and there is a need of protocol and pulse sequence standardization.

Most perfusion analysis remains observer-dependent ("eyeball" analysis) or dependent on bolus dispersion (semi-quantitative analysis). Fully quantitative analysis using CMR perfusion is currently time-consuming for clinical application. Perfusion CMR is an evolving field with numerous future directions.

\section{INTRODUCTION}

Cardiovascular disease is the main cause of death in most European countries, with a range of mortality rates between different countries (<3 per 1,000 inhabitants in the South-Western and Northern Europe and 9 per 1,000 inhabitants in Eastern Europe) $)^{(1)}$.

In patients with suspected coronary artery disease (CAD) exercise or pharmacological stress test are indicated for diagnostic work-up, as suggested by the recent European ${ }^{(2)}$ and American Guidelines ${ }^{(3)}$. As described by Nesto et al in the late 1980s, the ischemic cascade is a temporal sequence of hemodynamic, electrocardiographic abnormalities and symptoms ${ }^{(4)}$, and subendocardial and transmural perfusion defects are the first expression of the imbalance between myocardial oxygen supply and demand. Perfusion imaging, therefore, has a central role in investigation and management of patients with possible or actual coronary artery disease.

\section{MYOCARDIAL PERFUSION IMAGING}

Different non-invasive imaging modalities can evaluate myocardial perfusion by assessing the functional significance of epicardial coronary stenoses by demonstrating stress-induced ischemia (perfusion abnormalities) in coronary artery disease (CAD). Stress imaging techniques have advantages over the conventional exercise electrocardiogram, particularly superior diagnostic performance for detection of obstructive coronary disease and the ability to localize and quantify areas of ischemia ${ }^{(2)}$. When luminal obstruction is $<50 \%$, maximal flow during exercise can be maintained. Coronary stenosis of $>50 \%$ may be associated with ischemia as coronary blood flow becomes inadequate to meet cardiac metabolic demand during stress ${ }^{(5,6)}$. Although physical exercise is the most physiologic stimulus to induce myocardial hyperemia, pharmacological stress testing offers an effective alternative. Current clinically available techniques to evaluate myocardial perfusion are positron emission tomography (PET), single photon emission computed tomography (SPECT), myocardial contrast echocardiography (MCE), and recently cardiovascular magnetic resonance (CMR) Although PET is considered the gold standard technique, due to its ability to quantify absolute myocardial perfusion in $\mathrm{ml} / \mathrm{min} / \mathrm{g}$ of myocardium, this test is not widely available, it is expensive, and it uses ionising-radiation exposure. Its sensitivity and specificity for the detection of CAD is $79 \%$ and $85 \%$, respectively ${ }^{(7)}$.

SPECT is the most widely available myocardial perfusion test, it has been well validated and it has high sensitivity (mean values, 87-89\%) for detection of coronary artery disease but lower specificity (mean values, $73-75 \%)^{(2,8)}$. However, this imaging modality is limited by a considerable radiation burden, relatively poor spatial resolution (approximately $10 \mathrm{~mm}$ ), attenuation artefact caused by soft tissues, and long duration of the scan (4-6 hours for stress and rest scans). MCE can assess myocardial perfusion ${ }^{(9)}$ and has the advantages of easier access and affordability, but is significantly operator dependent and requires good echocardiographic windows. 
CMR perfusion imaging is an innovative and promising technique offering high spatial resolution $(2 \mathrm{~mm})$ without ionizing radiation exposure and its use is rapidly evolving in the diagnosis and management of patients with CAD. During a single scan of I hour duration, information can be gained on: I) regional and global myocardial function; 2) myocardial perfusion in regions supplied by normal or diseased arteries, both during stress and at rest; 3 ) the presence of intracavitary thrombus or microvascular obstruction in the setting of acute myocardial infarction (early gadolinium-enhancement imaging); 4) detection of myocardial infarction (late gadolinium-enhancement imaging); and 5) evaluation of myocardial viability ${ }^{(10)}$.

The first CMR perfusion study was described in 1987 by Johnston et al. (I) that reported the potential applications and limitations of gadolinium-diethylenetriamine pentaacetic acid (DTPA) as a myocardial perfusion agent in an animal model of coronary occlusion. Quantification of myocardial blood flow by first-pass myocardial perfusion imaging was consequently validated against microspheres ${ }^{(12,13)}$. The clinical feasibility of the technique in humans was demonstrated in the early 1990s by several groups ${ }^{(14,15)}$, and from this time the perfusion CMR literature has mainly been directed towards improving the technical robustness of the technique and validating it against other clinically available perfusion imaging modalities such as SPECT and PET.

This paper will review the role of CMR first-pass myocardial perfusion from the technical aspects of image acquisition and image analysis to the clinical applications in patients with coronary artery disease.

\section{CMR TECHNIQUE}

\section{Contrast agents}

Gadolinium-chelate contrast agents, such as Gd-DTPA, are extracellular paramagnetic contrast agents that accentuate the difference in tissue relaxation characteristics. Following intravenous administration, the contrast agent diffuses rapidly from the intravascular to the extracellular compartment. The corresponding increased image intensity is the result of the shortening of myocardial TI relaxation time caused by the contrast agent, and the magnitude of the signal enhancement depends on the amount of contrast agent present.

\section{Myocardial perfusion imaging}

Early studies in CMR myocardial perfusion with gadolinium-chelates showed that the overall image intensity is a function of several parameters, including the delivery and the dose of the contrast agent ${ }^{(16)}$. In general, lower doses cause lower signal to noise (SNR) in the myocardium, whereas higher doses determine a non-linear signal intensity in the left ventricular cavity (after contrast administration) resulting in an inaccurate input function. The optimal dose of contrast agent for perfusion CMR has not been determined, but a number of studies have helped identify the correct range. The best dose of gadodiamide for visual assessment of stress-induced regional perfusion abnormalities was identified with $0.1 \mathrm{mmol} / \mathrm{kg}$ bodyweight (as opposed to 0.05 and $0.15 \mathrm{mmol} / \mathrm{kg}$ bodyweight) using a GRE-EPI (Gradient Echo-Echo Planar Imaging) sequence ${ }^{(17)}$. Superior image quality was found with an SSFP (Steady State Free Precession) sequence with both dosage regimes of $0.05 \mathrm{mmol} / \mathrm{kg}$ and $0.025 \mathrm{mmol} / \mathrm{kg}$ bodyweight for $\mathrm{Gd}$ BOPTA, in comparison with TI-GRE or GRE-EPI sequences ${ }^{(18)}$. In a multicentre dose-ranging study it was established that the minimally efficacious dose of Gd-DTPA for detecting obstructive coronary artery disease was $0.05 \mathrm{mmol} / \mathrm{kg}$ (mean sensitivity $93 \pm 0 \%$, mean specificity $75 \pm 7 \%$ and mean accuracy $85 \pm 3 \%)^{(19)}$ when compared to 0.10 and $0.15 \mathrm{mmol} / \mathrm{kg}$. In this study, higher doses yielded greater myocardial enhancement but did not result in higher diagnostic accuracy. This is most likely due to the fact that higher contrast doses are more likely to cause susceptibility artefacts that could be mistakenly read as real perfusion defects. The same images were analysed using upslope analysis and a higher sensitivity and specificity was found at $0.1 \mathrm{mmol} / \mathrm{kg}$ than at $0.05 \mathrm{mmol} / \mathrm{Kg}$ (but again, lower specificity at $0.15 \mathrm{mmol} / \mathrm{kg})^{(20)}$.

A second important parameter is the injection rate of the contrast agent bolus. This has not been standardized yet and it varies between centres from $3.5 \mathrm{ml} / \mathrm{s}$ to $8 \mathrm{ml} / \mathrm{sec}$. In healthy myocardial segments, the myocardial enhancement is largely independent from the injection rate of the contrast agent bolus, as long as the injection speed is not below $4 \mathrm{ml} / \mathrm{s}^{(18)}$. This is consistent with Elkington et al. ${ }^{(21)}$ who demonstrated peak $\mathrm{Gd}$ concentration in the ascending aorta at an injection rate between 3 and $5 \mathrm{ml} / \mathrm{s}$.

\section{Myocardial viability imaging}

After the perfusion phase, the distribution of gadolinium in the heart is determined by the kinetics of access to different tissue types. Access (wash-in) to infarcted myocardium is slower, and therefore it has been demonstrated that late imaging at 10-20 minutes reveals high signal in infarcted tissue (increased volume of distribution), as gadolinium has washed out of normal myocardium. This has been validated histologically by $\mathrm{Kim}^{(22)}$. An improved inversion-recovery gradient-echo pulse sequence has the ability to produce a greater difference in signal intensity between normal and infarcted myocardium(23). Contrastenhanced MR allows the identification of myocardial scar and it is a validated method to assess myocardial viability. Other causes of abnormal gadolinium uptake are fibrosis or protein deposition in cardiomyopathy.

\section{Pulse sequences and acceleration factors}

The optimal sequence for myocardial perfusion CMR has not been determined. As described by Schwitter ${ }^{(24)}$, the available pulse sequences are: I) Turbo Fast Low-Angle Shot (FLASH), 2) Echo-Planar or Hybrid Echo-Planar (EPI) and 3) Steady-State Free Precession (SSFP).The ideal sequence for imaging this process should be very rapid, be able to "freeze" the cardiac motion, have a high SNR, high spatiotemporal 
resolution and should be robust to artefacts. The current available pulse sequences are briefly described as follows.

FLASH is the simplest method of rapid imaging. It collects one line of raw data per radiofrequency excitation, therefore being relatively slow and inefficient. However, it is considered a robust sequence because after each data readout the residual signal is discarded and gets a new signal for the next line. This sequence has an intrinsic poor SNR due to the low flip angle.

Hybrid EPI requires faster gradients and collects multiple lines of raw data after each radiofrequency pulse, and is effectively an accelerated variant of the FLASH technique. EPI is a faster sequence with a better SNR than FLASH. However, this sequence is prone to off-resonance artefacts. These can be reduced by running EPI faster, but at the cost of lower SNR.

TrueFISP has a higher SNR due to its higher flip angle. It is considered an efficient sequence because it re-uses residual magnetisation. However, the image acquisition is slower compared to the other sequences and, therefore, prone to motion artefacts as well as being more sensitive to off-resonance. Dark ring artefacts had been noted as more prominent with TrueFISP(25) (eyeball analysis).

Comparing the 3 sequences, hybrid EPI has the shortest time per image, which is important for minimizing cardiac motion artefacts. However, in the fastest version with minimal off-resonance errors, EPI has lower SNR than the other two sequences.

The increase in cardiac heart rate that often occurs during hyperemic stress may limit the acquisition window, resulting in incomplete myocardial coverage. Parallel acquisition methods such as sensitivity encoding (SENSE) are used to shorten data acquisition time ${ }^{(26)}$ also in $C M R^{(27)}$. The feasibility and high diagnostic accuracy of perfusion CMR with SENSE versus conventional angiography has been demonstrated in patients with $C A D^{(28)}$.

The hybrid-EPI is the sequence of choice to perform perfusion CMR, both in our centre and in other laboratories ${ }^{(29,30)}$. However, the other sequences are still widely used.

\section{Adenosine}

Adenosine is an endogenous nucleotide with potent vasodilator property in most vascular beds, except in renal afferent arterioles and hepatic veins (where it produces vasoconstriction). Adenosine exerts its pharmacological effects through activation of purine receptors, mainly $A_{1}$ - and $A_{2}$-adenosine receptors: peripheral vasodilation is the effect of the $A_{2}$-receptor agonism whereas the negative chronotropic, dromotropic and inotropic effects on the heart are due to $A_{1}$-receptor activation.
Normal resting flow in the coronary arteries is approximately $1 \mathrm{ml} / \mathrm{min} / \mathrm{g}$ of myocardium. The coronary hyperemia caused by adenosine determines an increase in perfusion in normal coronary arteries reaching 3-4 $\mathrm{ml} / \mathrm{min} / \mathrm{g}$, whereas in myocardial territories supplied by stenotic vessels perfusion is restricted to resting values of $1 \mathrm{ml} / \mathrm{min} / \mathrm{g}$ (the vessel is already maximally dilated at rest to overcome the limited blood flow caused by the stenosis) ${ }^{(31)}$. Adenosine causes heterogenous myocardial perfusion and this can be detected by perfusion CMR. It can also cause ischemia through a transmural "steal phenomenon".

Adenosine is infused at the rate of $140 \mu \mathrm{g} \cdot \mathrm{Kg}_{-}{ }^{-} \cdot \mathrm{min}^{-}{ }^{-}$using a syringe pump. CMR first-pass perfusion acquisition starts 3 minutes after the beginning of adenosine infusion. The safety profile of adenosine in the context of stress perfusion imaging was assessed in 9,256 consecutive patients undergoing radionuclide imaging ${ }^{(32)}$. Adenosine infusion is safe, and its vasodilator and negative dromotropic effects are generally well tolerated. Serious side effects are relatively rare, and they rapidly reverse with termination of the infusion, due to adenosine's ultrashort half-life $(<10$ seconds). However, caution should be used because there are some reports of the proarrhthymic effects of adenosine ${ }^{(33)}$.

Dipyridamole is an alternative drug, which increases adenosine through indirect mechanisms. It is actively metabolized in the liver and, therefore, its vasodilator capacity is variably dependent on individual metabolism rate. Compared to adenosine, dypiridamole has a longer half-life (30 minutes), and prolonged side effects. Typically, the infusion dose is $0.56 \mathrm{mg} \cdot \mathrm{Kg}^{-1} \cdot \mathrm{min}^{-1}$ for 4 minutes and the images acquisition starts after 6 minutes. Compared to dypiridamole, adenosine has been shown to lower costs both for patients' monitoring and adverse events management ${ }^{(34)}$.

\section{Adenosine or dobutamine stress CMR perfusion?}

Adenosine has a more favorable safety profile and is well established for the assessment of myocardial perfusion, both with echocardiography and nuclear cardiac imaging.

In 79 consecutive patients with suspected or known CAD (but no history of myocardial infarction) the diagnostic value of inducible wal motion abnormalities during dobutamine or adenosine stress CMR were compared with invasive coronary angiography ${ }^{(35)}$. Dobutamine showed to be superior (sensitivity: $89 \%$, specificity: 80\%) for the detection of inducible wall motion abnormalities compared to adenosine (sensitivity: 40\%, specificity: 96\%) in patients with epicardial coronary stenosis $>50 \%$ luminal narrowing. Adenosine stress CMR wall motion was highly specific, because it identified only patients with high-grade coronary stenosis ( $>75 \%$ luminal narrowing). Visual assessment of adenosine stress perfusion defects had a good sensitivity but lower specificity ( $91 \%$ and $62 \%$, respectively) ${ }^{(35)}$. 


\section{Imaging protocol}

Typically, 3 short-axis slices (basal, mid-cavity and apical) are acquired both during stress and at rest, in order to provide complete myocardial segmental coverage, according to the recommended 17-segment myocardial model for the tomographic imaging of the heart ${ }^{(36)}$. And although there is variability in the coronary blood supply to myocardial segments, the 17 myocardial segments are assigned to one of the three major coronary arteries ${ }^{(36)}$. The 3 short axis images are acquired throughout the cardiac cycle and triggered to the R wave on the ECG tracing; approximately 40-60 images for each slice location. Breathing should be suspended for at least the early part of the image acquisition (during the first pass) to minimize breathing motion artefacts and facilitate diagnostic interpretation. The short-axis planes can evaluate only 16 myocardial segments, failing to assess the ventricular apex. A combined long- and short-axis imaging protocol has been proposed; however, the long-axis views were not able to depict inducible perfusion defects in the apex, despite apical ischemia being demonstrated with recent SPECT in the same patients ${ }^{\left({ }^{37}\right)}$. The number of short-axis slices is strictly limited by the patient's heart rate, i.e. bradycardia could allow the acquisition of extra slices. Fifty cardiac cycles are acquired to allow the complete track of the dynamic process of contrast bolus from the moment of the peripheral injection to the diffusion in the myocardium of the left ventricle. Probably only 30 cardiac cycles are needed, but the exact arrival time of the bolus is unpredictable.

Resting perfusion images are acquired 20 minutes after the stress perfusion images, a time window to allow partial contrast wash-out from the previous injection.

\section{Interpretation algorithm: stress-rest or stress-late gadolinium enhancement}

With CMR, the rest imaging assesses the resting perfusion, and the stress imaging shows myocardial perfusion during hyperemia. This is in distinction to perfusion SPECT where the both rest and stress imaging are strongly modulated by myocardial viability. Therefore, the stress-rest protocol in CMR resembles more the PET approach to calculate the perfusion reserve. An alternative approach combines myocardial perfusion at stress to assess myocardial ischemia and late-gadolinium enhancement to probe myocardial viability ${ }^{(38)}$. The reported sensitivity, specificity and accuracy of the combined diagnostic approach was $89 \%$, $87 \%$ and $88 \%$, respectively compared to perfusion CMR alone (84\%,58\% and $68 \%$, respectively). The ideal protocol requires further research, but for simplicity the stress/late enhancement approach is the most attractive.

\section{Image analysis}

The measurement of absolute tissue perfusion by CMR remains a more difficult but very desirable goal. Whether the efforts to apply quantitative analysis provide significantly improved accuracy of the technique or whether it helps only to ameliorate the variability and bias of less experienced readers is still unclear ${ }^{(39)}$. Even though this argument has been made for cardiac nuclear studies, it may well be applied to CMR first-pass perfusion imaging. Analysis is either visual to identify areas of low signal representing reduced myocardial perfusion, or quantitative with dedicated computer software.

\section{Qualitative analysis}

A rapid visual assessment is considered more appropriate for a clinical application of the imaging technique. Perfusion defects are defined as focal areas of diminished myocardial enhancement, which therefore appear hypointense (darker) compared to normal segments (Figure I). In a study comparing the perfusion CMR findings with angiography, the sensitivity and specificity of visual assessment was 93\% and 60\%, respectively ${ }^{(40)}$. Qualitative MR reporting was possible in $98 \%$ of the cases.As for other imaging techniques, the qualitative approach is observerdependent and potentially less accurate than the quantitative analysis.

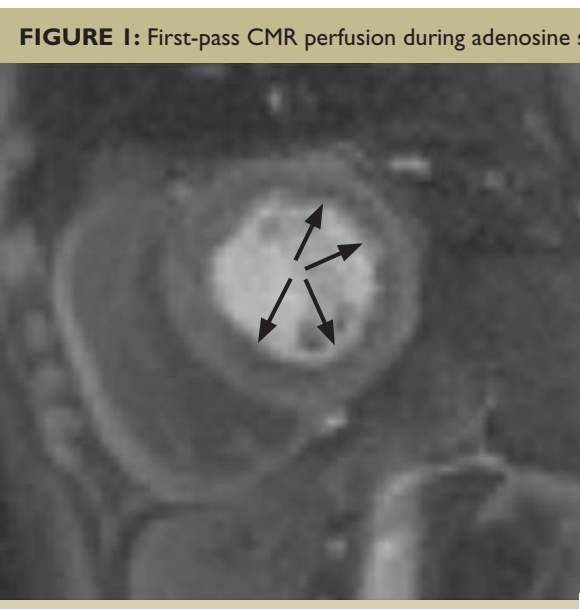

CASE A: subendocardial perfusion defect in the lateral and inferior walls extending to the inferior septum; subsequent coronary angiography showed stenosis of the mid right coronary artery and proximal left circumflex coronary artery.

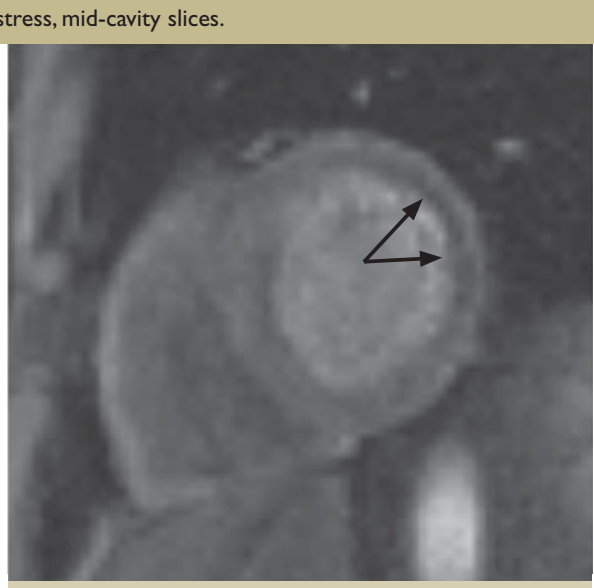

CASE B: subendocardial perfusion defect in the lateral wall; coronary angiography confirmed $90 \%$ stenosis of the first obtuse marginal branch.

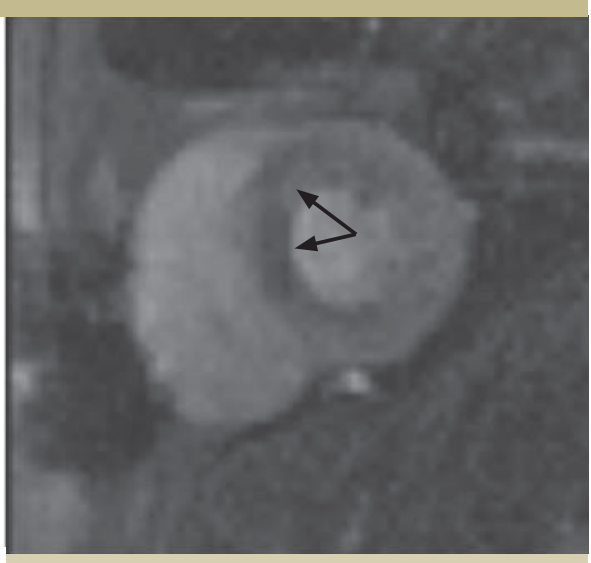

CASE C: extensive perfusion defect in the anterior septum; coronary angiography showed $85 \%$ stenosis of the mid left anterior descending coronary artery. 


\section{Quantitative analysis}

Quantitative analysis aims to remove dependence on observer and on bolus dispersion.

Myocardial perfusion reserve (MPR) was proposed since the early 1970s as an index of functional severity of coronary stenosis ${ }^{(41,42)}$, and it is calculated as the ratio of hyperemic and baseline myocardial blood flow. In humans, PET is the only validated imaging method to measure quantitative myocardial blood flow and MPR ${ }^{(43)}$. Cullen et al. demonstrated the feasibility of performing myocardial perfusion reserve index measurement by first-pass CMR in normal volunteers and patients with $C A D^{(44)}$ compared to quantitative angiography and PET data. Similar reliable assessment of MPR index by CMR perfusion in a clinical context was reported by other groups ${ }^{(45)}$. Some preliminary steps need to be taken before performing the quantitative analysis: data should be corrected for respiratory motion and coil sensitivity, then signal intensities should be converted into contrast agent concentration in order to allow for input correction.

For the analysis, the left ventricle is divided into regions of interest (ROI), according to the 17-segments model(36). Endocardial and epicardial contours are traced on each image and the signal intensity over time (upslope), arrival time, time to peak signal, mean transit time (MTT) are calculated for each ROI. Then different models may be applied to calculated perfusion(46): fully quantitative or semi-quantitative analysis. The latter aims to remove operator-dependence, whereas the fully quantitative analysis removes dependence on bolus dispersion as well.

For the semi-quantitative analysis the upslope of the signal-intensity curve is the most widely used parameter, being also the most sensitive parameter to differences in myocardial blood flow ${ }^{(46)}$. Additionally, it correlates closely with microspheres (in dogs) ${ }^{(47.48)}$, and PET measurements (in humans) ${ }^{(49)}$. An underestimation of coronary flow reserve by CMR perfusion measurements compared to PET was reported by Ibrahim et al. in patients with angiographically documented $C A D^{(50)}$. The upslope index yielded a sensitivity, specificity and diagnostic accuracy of $69 \%, 89 \%$ and $79 \%$, respectively, for the localization of coronary artery stenosis $(>75 \%, M P R<1.2)$ and a sensitivity, specificity and diagnostic accuracy of $86 \%, 84 \%$ and $85 \%$, respectively, for detection of reduced coronary flow reserve $(\mathrm{PET}<2.0, \mathrm{MPR}<1.3)$.

The signal intensity (SI) curve is a combination of perfusion and diffusion, both of which are influenced by blood flow ${ }^{(51)}$. The early phase of SItime curve is mainly influenced by perfusion and to a lesser extent by diffusion, and the latter parts are increasingly influenced by diffusion. Both the wash-in and wash-out are strongly influenced by the compactness of the contrast bolus, and for a reliable calculation it should be small and compact. Ischemic myocardial segments show a slower passage of the contrast agent ${ }^{(52)}$, (lower peak myocardial signal intensity, slower rate of signal increase and longer time to peak perfusion), resulting in a stronger influence of diffusion and a less pronounced or even nonexistent downslope. A linear fit of the upslope of the signal intensity-time curve was proposed by Al-Saadi as a new and easy method for the determination of $\mathrm{MPR}^{(53)}$ and to minimize the influence of diffusion. This parameter was demonstrated to be highly reproducible, with good interobserver and intraobserver variability.

Fully quantitative analysis can be achieved by normalized upslope or ful deconvolution analysis ${ }^{(54)}$ constrained by a mathematical model of contrast agent kinetics. A requirement for quantitative perfusion is to maintain a linear relationship between $\mathrm{SI}$ and contrast concentration (maintained only by low contrast concentration) ${ }^{(46)}$, especially for the arterial input function.

However, the improvement of contrast to noise ratio is dependent on the use of higher contrast agent dose. In order to overcome this problem, Christian et al. ${ }^{(47)}$ introduced the dual-bolus injection technique that allows measurement of the input function with a low-dosage contrast injection, followed by the measurement of myocardial contrast enhancement with a higher bolus dosage. Hsu and colleagues reported that, in healthy human subjects, rest and stress myocardial blood flow and MPR evaluated by dual-bolus perfusion MRI were similar to published data, whereas semiquantitative methods such as the upslope index were significantly underestimating $M \mathrm{PR}^{(54)}$. With a new method of assessing arterial input function, myocardial perfusion reserve (MPR) can also be achieved by a single bolus technique with a high dose gadolinium ${ }^{(55)}$.

Overall, first-pass perfusion CMR can feasibly evaluate the subendocardial layer and assess MPR; however, this qualitative approach can be time-consuming and not always reproducible.

\section{Clinical applications}

Clinical applications of CMR perfusion have been described since the year 2000. Al-Saadi evaluated 15 patients with single-vessel CAD and 5 controls without significant CAD using first-pass perfusion $\mathrm{CMR}^{(53)}$. He concluded that the technique yielded a high sensitivity, specificity and diagnostic accuracy $(90 \%, 83 \%$ and $87 \%$, respectively). Interobserver and intraobserver variability were low ( $r=0.96$ and 0.99$)$. Schwitter et al. assessed myocardial perfusion in patients with CAD and healthy subjects comparing perfusion CMR, PET and coronary angiography ${ }^{(49)}$ The receiver-operator characteristic curve for the subendocardial 
upslope showed that perfusion CMR had a high sensitivity (91\%) and specificity (94\%) for CAD defined by PET (coronary flow reserve $<1.65$ in $\geq$ I segment). Similarly, when CAD was assessed by quantitative coronary angiography (diameter stenosis $\geq 50 \%$ in $\geq$ I artery), perfusion CMR demonstrated a sensitivity of $87 \%$ and a specificity of $85 \%$. The authors concluded that perfusion CMR may qualify for clinical application in the management of CAD because it provides important information on the amount of compromised myocardium, even when perfusion abnormalities are confined to the subendocardial layer.

Adenosine perfusion CMR has also demonstrated the ability to detect subendocardial perfusion defects in patients with cardiac syndrome $X^{(56)}$. In 10-matched control patients, myocardial perfusion increased in both the endocardium and the epicardium with adenosine.

Recently, adenosine CMR perfusion demonstrated a high negative predictive value in patients presenting to an emergency department with chest pain, negative troponin and non-diagnostic electrocardiogram ${ }^{(57)}$. None of the subjects with normal adenosine stress CMR scan were diagnosed with an adverse cardiac event after I-year follow-up.

\section{FUTURE DIRECTIONS}

Selective adenosine $A_{2 A}$ receptor agonists currently in development may play an important role for cardiac perfusion imaging. Initial clinical experiences reported this new agent as effective as adenosine in detecting and quantifying perfusion defects with a concomitant reduction in incidence and severity of side effects and simplicity of bolus administration ${ }^{(58,59)}$. In addition, the simplified logistics of the drug delivery (i.e. no need of infusion pump and long tubing to reach the patient in the magnet) and the potential use in asthmatic patients make this new agent represent an attractive alternative.

Limited data are available regarding the application of intravascular gadolinium-based contrast media. Intravascular contrast media yield lower signal intensity in the myocardium than extracellular contrast agents because of reduced exchange into the extracellular space during the first pass ${ }^{(24)}$. Dose effects on peak signal enhancement have been described in inversion recovery EPI using intra- and extravascular gadolinium-based contrast agents( ${ }^{(60)}$. Jerosch-Herold et al. have demonstrated that perfusion index with an intravascular contrast agent (MS-325) is accurately estimated if corrected by the time-tomaximum upslope ${ }^{(61)}$. Currently, the value of quantitative perfusion with intravascular contrast agent in humans with CAD is not known.

A recent paper by Saeed and coworkers described the combination of intravascular and extracellular Gd-chelates in discriminating acute from chronic infarctions on late enhancement images in an experimental animal model(62). There was no late enhancement produced by intravascular Gd-chelate in chronic infarction, likely due to poor vascularization and perfusion in scar tissue.

The role of 3 Tesla magnets for myocardial perfusion remains to be evaluated. Some early investigations of $3 T$ versus $1.5 T$ are showing the predictable benefit of the higher SNR at $3 \mathrm{~T}^{(63)}$.

As in SPECT, an agent that could directly assess areas of ischemia which persist after stress is highly desirable for CMR, so that first-pass imaging is not needed. Manganese is a paramagnetic ion with pharmacokinetic properties similar to those of $99 \mathrm{mTC}$-sestamibi and there are experimental studies with manganese-enhanced $C M R^{(64)}$. However, the cardiotoxic and cardio depressant effects of manganese ${ }^{(65)}$ make its clinical application not feasible at this time.

Blood oxygen level dependent (BOLD) CMR is an interesting technique with the potential to identify areas of myocardial ischemia ${ }^{(66,67)}$, as signal changes within the myocardium based on changes in deoxyhaemoglobin, without using contrast media ${ }^{(68)}$. However, large comparative studies with gold standard methods of myocardial ischemia are required.

\section{CONCLUSIONS}

Perfusion CMR is an attractive imaging modality, which is becoming comparable with other clinically diagnostic tests. SPECT and PET are well clinically validated and have good accuracy for detection of significant CAD. However, these techniques have a rather low spatial resolution and are not suitable for the detection of subendocardial perfusion defect. In addition, the radiation burden, the potential for attenuation artefacts (SPECT) and the limited availability (PET) are limitations of these imaging techniques. An integrated assessment of myocardial perfusion, function and viability is feasible with CMR. In addition, compared to other clinically available imaging techniques, CMR perfusion has excellent spatial resolution and no ionising radiation exposure. However, it is not widely available and there is a need of protocol and pulse sequence standardization.

Most perfusion analysis remains observer-dependent ("eyeball" analysis) or dependent on bolus dispersion (semi-quantitative analysis). Fully quantitative analysis using CMR perfusion is currently time-consuming for clinical application.

Perfusion CMR is an evolving field with numerous future directions. 


\section{REFERENCES:}

1. Scholte op ReimerWJM, GittAK, Boersma E, Simoons ML (eds.). Cardiovascular Disease in Europe. Euro Heart Survey and National Registries of Cardiovascular Diseases and Patient Management 2004. Sophia Antipolis; European Society of Cardiology 2004.

2. Fox K, Alonso Garcia MA, Ardissino D et al. Guidelines of the management of stable angina pectoris: exective summary: the Task Force on the Management of Stable Angina Pectoris of the European Society of Cardiology. Eur Heart J 2006;27( I I): | 34 | - | 38 |

3. Gibbons RJ, Abrams J, Chatterjee K et al. ACC/AHA 2002 guideline update for the management of patients with chronic stable angina-summary article. J Am Coll Cardiol 2003;4I(1):159-168.

4. Nesto RW, Kowalchuk G]. The ischemic cascade: temporal sequence of hemodynamic, electrocardiographic and symptomatic expressions of ischemia. Am J Cardiol 1987;59(7):23C-30C.

5. Gould KL, Kirkeeid RL, Buchi M. Coronary flow reserve as a physiologic measure of stenosis severity. Am Coll Cardiol 1990; 15:459-474.

6. Gould KL. Effects of coronary stenoses on coronary flow reserve and resistance. Am J Cardiol 1974:34:48-55.

7. Laubenbacher C, Rothley J, Sitomer K, et al. An automated analysis program for the evaluation of cardiac PET studies: initial results in the detection and localization of coronary artery disease using N-13 ammonia. J Nucl Med 1993;34:968-78.

8. Klocke FJ, Baird MG, Lorell BH et al. ACC/AHA/ASNC guidelines for the clinical use of cardiovascular radionuclide imaging-an executive summary: a report of the American College of Cardiology/American Heart Association Task Force on Practice Guidelines (ACC/AHA/ASNC Committee to Revise the 1995 Guidelines for the Clinical Use of Cardiac Radionucleide Imaging). Circulation 2003; I08(I I): | 404- 18

9. Lang R, Mor-Avi. Clinical utility of contrast-enhanced echocardiography. Clin Cardiol 2006;29(suppl I):II5-25.

10. Pennell DJ.Cardiovascular magnetic resonance and the role of adenosine pharmacological stress. Am J Cardiol 2004;94(suppl):26D-32D.

11. Johnston DL, Liu P, Lauffer RB, Wedeen VJ, Rosen BR, Brady TJ, Okada RD. Use of gadolinium-DTPA as a myocardial perfusion agent: potential applications and limitations for magnetic resonance imaging.J Nucl Med 1987;28(5):87|-7.

12. Wilke N, Simm C, Zhang J, Ellermann J,Ya X, Merkle H, Path G, Ludemann H, Bache RJ Ugurbil K. Contrast-enhanced first-pass myocardial perfusion imaging: correlation between myocardial blood flow in dogs at rest and during hyperemia. Magn Reson Med 1993;29(4):485-497.

13. Wilke N, Jerosch-Herold M, Wang Y, Huang Y, Christensen BV, Stillman AE, Ugurbil K, McDonald K, Wilson RF. Myocardial perfusion reserve: assessment with multisection quantitative, first-pass MR imaging. Radiology 1997;204(2):373-384

14. Atkinson DJ, Burnstein D, Edelman RR. First-pass cardiac perfusion: evaluation with ultrafast MR imaging. Radiology 1990; 174 (3Part I):757-62.

I5. Van Rugge FP, Boreel JJ,Van der Wall EE, Van Dijkman PR, Van der Laarse A, Doornbos J, De Roos A, Den Boer JA, Bruschke AV, Van Voorthuisen AE. Cardiac first-pass and myocardial perfusion in normal subjects assessed by sub-second Gd-DTPA enhanced MR imaging. J Comput Assist Tomogr 1991;15(6):959-65.

16. Burstein D, Taratuta E, Manning WJ. Factors in myocardial "perfusion" imaging with ultrafast MRI and Gd-DTPA administration. Magn Reson Med 1991; 20(2):299-305.

17. Paetsch I, Foll D, Langreck H, Herkommer B, Klein C, Schalla S, Fleck E, Nagel E. Myocardial perfusion imaging using OMNISCAN: a dose finding study for visual assessment of stress-induced regional perfusion abnormalities. J Cardiovasc Magn Reson 2004:6(4):803-809.

18. Gebker R, Paetsch I, Neuss M, Schnackenburg B, Bornstedt A, Jahnke C, Gomaa O, Fleck E, Nagel E. Determinants of myocardial response in CMR perfusion imaging using Gd-BOPTA (Multihance). J Cardiovasc Magn Reson 2005;7(3):565-572.

19. Wolff SD, Schwitter J, Coulden R, Friedrich MG, Bleumke DA, Biederman RW, Martin ET, Lansky AJ, Kashanian F, Foo TKF, Licato PE, Comeau CR. Myocardial first-pass perfusion magnetic resonance imaging. A multicenter dose-ranging study. Circulation 2004; I 10:732-737

20. Giang TH, Nanz D, Coulden R, Friederich M, Graves M, Al-Saadi N, Luscher TF, Von Schulthess GK, Schwitter J. Detection of coronary artery disease by magnetic resonance myocardial perfusion imaging with various contrast medium dose: first European multi-center experience. Eur Heart J 2004;25 ( I 8): I 657-65.

21. Elkington AG, He T, Gatehouse PD, Prasad SK, Firmin DN, Pennell D). Optimization of the arterial input function for myocardial perfusion cardiovascular magnetic resonance. J Magn Reson Imaging 2005;2I (4):354-359.

22. Kim RJ, Fieno DS, Parrish TB et al. Relationship of MRI delayed contrast enhancement to irreversible injury, infarct age, and contractile dysfunction. Circulation 1999: 100:1992-2002.
23. Simonetti OP, Kim RJ, Fieno DS, et al. An improved MR imaging technique for the visualization of myocardial infarction. Radiology 200 1; 1 8:215-223.

24. Schwitter J. Myocardial perfusion. J Magn Reson Imaging 2006;24:953-963.

25. Fenchel M, Helber U, Simonetti OP, Stauder NI, Kramer U, Nguyen CN, Finn JP, Claussen $C D$, Miller $S$. Multislice first-pass myocardial perfusion imaging: comparison of saturation recovery (SR)-TrueFISP-two dimensional (2D) and SR-TurboFLASH-2D pulse sequences. J Magn Reson Imaging 2004;19(5):555-563.

26. Pruessmann KP,Weiger M, Scheidegger MB, Boesiger P. SENSE: sensitivity encoding for fast MRI. Magn Reson Med 1999;42:952-962.

27. Preussmann KP, Weiger M, Boesiger P. Sensitivity encoded cardiac MRI. J Cardiovasc Magn Reson 2001:3(1): 1-9.

28. Plein S, Radjenovic A, Ridgway JP, Barmby D, Greewood JP, Ball SG, Sivananthan MU Coronary artery disease: myocardial perfusion MR imaging with sensitivity encoding versus conventional angiography. Radiology 2005;235:423-430.

29. Hsu LY, Rhoads KL, Holly JE, Kellman P, Aletras AH, Arai AE. Quantitative myocardia perfusion analysis with a dual-bolus contrast-enhanced first-pass MRI technique in humans. J Magn Reson Imaging 2006:23(3):315-322.

30. Kim D, Axel L. Multislice, dual-imaging sequence for increasing the dynamic range of the contrast-enhanced blood signal and CNR of myocardial enhancement at 3T. J Magn Reson Imaging 2006;23(I):81-86.

31. Wilson RF, Wyche K, Christensen BV, Zimmer S, Laxson DD. Effects of adenosine on human coronary arterial circulation. Circulation 1990:82(5): 1595-1606.

32. Cerqueira MD,Verani MS, Schwaiger M, Heo J, Iskandrian AS. Safety profile of adenosine stress perfusion imaging: results from the Adenoscan MulticenterTrial Registry. J Am Col Cardiol 1994:23(2):384-389.

33. Mallet ML. Proarrhythmic effects of adenosine: a review of the literature. Emerg Med 2004;21:408-410.

34. Hilleman DE, Lucas BD Jr. Mohiuddin SM, Holmberg MI. Cost-minimization analysis of intravenous adenosine and dypiridamole in thallous chloride TI 201 SPECT myocardial perfusion imaging. Ann Pharmacother 1997;31:974-979.

35. Paetsch I, Jahnke C, Wahl A, Gebker R, Neuss M, Fleck E, Nagel E. Comparison of dobutamine stress magnetic resonance, adenosine stress magnetic resonance, and adenosine stress magnetic resonance perfusion. Circulation 2004; I I0(7):835-842.

36. Cerqueira MD, Weissman NJ, Dilsizian V, Jacobs AK, Kaul S, Laskey WK, Pennell DJ, Rumberger JA, Ryan T, Verani MS; American Heart Association Writing Group on Myocardial Segmentation and Registration for Cardiac Imaging. Circulation 2002; 105(4):539-542

37. Elkington AG, Gatehouse P, Prasad SK, Moon JC, Firmin DN, Pennell DJ. Combined longand short-axis myocardial perfusion cardiovascular magnetic resonance. J Cardiovas Magn Reson 2004;6(4):811-816.

38. Klem I, Heitner JF, Shah DJ, Sketch MH Jr, Behar V, Weinsaft J, Cawley P, Parker M, Elliott M, Judd RM, Kim RJ. Improved detection of coronary artery disease by stress perfusion cardiovascular magnetic resonance with the use of delayed enhancement infarction imaging. J Am Coll Cardiol 2006;47(8): 1630-1638.

39. Wackers FJ. Science, art, and artefacts: how important is quantification for the practising physician interpreting myocardial perfusion studies? I Nucl Cardiol 1994; I (5Pt 2):SI09-117.

40. Sensky PR, Samani NJ, Reek C, Cheeryman GR. Magnetic resonance perfusion imaging in patients with coronary artery disease: a qualitative approach. Int J Cardiovasc Imaging 2002; | 8(5):373-383.

4I. Gould KL, Lipscomb K, Hamilton GW. Physiologic basis for assessing critical coronary stenosis: instantaneous flow response and regional distribution during coronary hyperemia as measure of blood flow reserve. Am J Cardiol 1974:33:87-94.

42. Gould KL, Lipscomb K. Effects of coronary stenoses on coronary flow reserve and resistance. Am J Cardiol 1974:34:48-55.

43. Shelton ME, Senneff MJ, Ludbrook PA, Sobel BE, Bergmann SR. Concordance of nutritive myocardial perfusion reserve and flow velocity reserve in conductance vessels in patients with chest pain with angiographically normal coronary arteries. J Nucl Med 1993:34(5):717-722

44. Cullen JHS, Horsfield MA, Reek CR, Cherryman GR, Barnett DB, Samani NJ.A myocardia perfusion reserve index in humans using first-pass contrast-enhanced magnetic resonance imaging. J Am Coll Cardiol 1999;33: | 386- 1394

45. Al-Saadi N, Nagel E, Gross M, Schnackenburg B, Paetsch I, Klein C, Fleck E. Improvement of myocardial perfusion reserve after coronary intervention: assessment with cardiac magnetic resonance imaging. J Am Coll Cardiol 2000;36: I557-I564. 
46. Jerosh-Herold M, Teja Seethamraju A, Swingen CM, Wilke NM, Stillman AE. Analysis of myocardial perfusion MRI. J Magn Reson Imaging 2004; 19:758-770.

47. Christian TF, Rettmann DW, Aletras AH, et al. Absolute myocardial perfusion in canines measured by using dual-bolus first-pass MR imaging. Radiology 2004;232:677-684.

48. Klocke FJ, Simonetti OP, Judd RM, Kim RJ, Harris KR, Hedjbeli S, Fieno DS, Miller S, Chen V, Parker MA. Limits of detection of regional differences in vasodilated flow in viable myocardium by first-pass magnetic resonance perfusion imaging. Circulation 2001; 104(20):2412-2416.

49. Schwitter J, Nanz D, Kneifel S, Bertschinger K, Buchi M, Knusel PR, Marincek B, LuscherTF,Von Shulthness GK. Assessment of myocardial perfusion in coronary artery disease by magnetic resonance: a comparison with positron emission tomography and coronary angiography. Circulation 200 I; 103:2230-2235.

50. Ibrahim T, Nekolla SG, Schreiber K, Odaka K, Volz S, Mehilli J, Guthlin M, Delius W. Schwaiger M. Assessment of coronary flow reserve: comparison between contrastenhanced magnetic resonance imaging and position emission tomography. J Am Coll Cardiol 2002;29:864-870.

5।. Tong CY, Prato FS, Wisenberg G, Lee TY, Carroll E, Sandler D, Wills J, Drost D. Measurements of the extraction efficiency and distribution volume for Gd DTPA in normal and diseased canine myocardium. Magn Reson Med 1993;30:337-346.

52. Uren NG, Melin JA, Bruyne B, Wijns W, Baudhiun T, Camici PG. Relation between myocardial blood flow and the severity of coronary artery stenosis. N Engl J Med 1994:330:1782-1788

53. Al-Saadi, Nagel E, Gross M, Bornstedt A, Schnackenburg B, Klein C, Klimek W, Oswald $\mathrm{H}$. Fleck E. Noninvasive detection of myocardial ischemia from perfusion reserve based on cardiovascular magnetic resonance. Circulation 2000; 10 1:1379- I 383.

54. Hsu LY, Rhoads KL, Holl JE, Kellman P, Aletras AH, Arai AE. Quantitative myocardial perfusion analysis with a dual-bolus contrast-enhanced first-pass MRI technique in humans. J Magn Reson Imaging 2006;23:315-322.

55. Gatehouse P, Elkington AG, Ablitt NA, Yang GZ, Pennell DJ, Firmin DN. Accurate assessment of the arterial input function during high-dose myocardial perfusion cardiovascular magnetic resonance. J Magn Reson Imaging 2004:20(I):39-45.

56. Painting JR, Gatehouse PD, Yang GZ, Grothues F, Firmin DN, Collins P, Pennell D. Abnormal subendocardial perfusion in cardiac syndrome $X$ detected by cardiovascular magnetic resonance imaging. N Eng J Med 2002;346: 1948- 1953.

57. Ingkanisorn WP, Kwong RY, Bohme NS, Geller NL, Rhoads KL, Dyke CK, Paterson DI, Syed MA, Aletras AH, Arai AE. Prognosis of negative adenosine stress magnetic resonance in patients presenting to an emergency department with chest pain. I Am Coll Cardiol 2006;47:1427-32.

58. Hendel RC, Bateman TM, Cerqueira MD, Iskandrian AE, Leppo JA, Blacjburn B, Mahmarian JJ. Initial clinical experience with regadenoson, a novel selective adenosine A2A agonist for pharmacologic stress single-photon emission computed tomography myocardial perfusion imaging. J Am Coll Cardiol 2005:46( ( I):2069-2075.

59. Udelson JE, Heller GV, Wackers F], Chai A, Inchman D, Coleman PS, Dilsizian V, DiCarli M, Hachamovitch R, Johnson JR, Barrett RJ, Gibbons RJ. Randomized, controlled dose-ranging study of selective adenosine A2A receptor agonist binodenoson for pharmacological stress as an adjunct to myocardial perfusion imaging. Circulation 2004; 109:457-464

60. Wendland MF, Saeed M, Yu KK, Roberts TP, Lauerma K, Derugin N, Varadarajan Watson $A D$, Higgins $C B$. Inversion recovery EPI of bolus transit in rat myocardium using intravascular and extravascular gadolinium-based MR contrast media: dose effects on peak signal enhancement. Magn Reson Med 1994;32(2):319-329.

6I. Jerosch-Herold M, Hu X, Murthy NS, Rickers C, Stillman AE. Magnetic resonance imaging of myocardial contrast enhancement with MS-325 and its relation to myocardial blood flow and the perfusion reserve. J Magn Reson Imaging 2003; I 8(5):544-554.

62. Saeed M,Weber O, Lee R, Do L, Martin A, Saloner D, Ursell P, Robert P, Corot C, Higgins C. Discrimination of myocardial acute and chronic (scar) infarction on delayed contrastenhanced mangnetic resonance imaging with intravascular magnetic resonance contrast media. J Am Coll Cardiol 2006;48:1961-1968.

63. Araoz PA, Glockner JF, McGee KP, Potter DD Jr, Valeti VU, Stanley DW, Christian TF. 3Tesla MR imaging provides improved contrast in first-pass myocardial perfusion imaging over a range of gadolinium doses. J Cardiovasc Magn Reson 2005;7(3):559-564.

64. Natanzon A, Aletras AH. Hsu LY, Arai AE. Determing canine myocardial area at risk with manganese-enhanced MR imaging. Radiology 2005;236:859-866.

65. Wolf GL, Baum L. Cardiovascular toxicity and tissue proton TI response to manganese injection in the dog and rabbit. AJR Am J Roentgenol 1983; 141:193-197.
66. Fieno DS, Shea SM, LiY, Harris KR, Finn JP. Li D. Myocardial perfusion imaging based on the blood oxygen level-dependent effect using T2-prepared steady-state free-precession magnetic resonance imaging. Circulation 2004; I I0( I0): I 284- 1290.

67. Egred M, Waiter GD, Al-Mohammad A, Semple ST, Redpath TW, Walton S. Blood oxygen level dependent (BOLD) MRI: a novel technique for the detection of myocardial ischemia. Eur J Intern Med 2006; 17(8):55I-555.

68. Li D, Dhawale P, Rubin PJ, Haacke EM, Gropler RJ. Myocardial signal response to dipyridamole and dobutamine: demonstration of the BOLD effect using a double-echo gradient-echo sequence. Magn Reson Med 1996;36(1): 16-20. 